\title{
Celiac disease-specific and inflammatory bowel disease-related antibodies in patients with recurrent aphthous stomatitis
}

\author{
Borivoj Bijelića,*, Ivana Z. Matić ${ }^{\mathrm{b}}$, Irina Besu ${ }^{\mathrm{b}}$, Ljiljana Jankovića ${ }^{\mathrm{a}}$ Zorica Juranić ${ }^{\mathrm{b}}$, \\ Senka Marušićc , Sladjana Andrejević ${ }^{\mathrm{d}}$ \\ ${ }^{a}$ School of Dental Medicine, University of Belgrade, dr Subotica 4, Belgrade 11000, Serbia \\ ${ }^{\mathrm{b}}$ Institute of Oncology and Radiology of Serbia, Pasterova 14, Belgrade 11000, Serbia

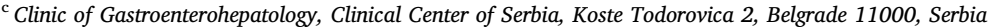 \\ ${ }^{\mathrm{d}}$ Clinic of Allergy and Clinical Immunology, Clinical Center of Serbia, Koste Todorovica 2, Belgrade 11000, Serbia
}

\section{A R T I C L E I N F O}

\section{Keywords:}

Recurrent aphthous stomatitis

Celiac disease

Inflammatory bowel disease

\begin{abstract}
A B S T R A C T
The etiology of recurrent aphthous stomatitis (RAS) remains unknown. RAS can be presented as primary, idiopathic condition and as a secondary RAS, which is associated with a systemic disease. The aim of our study was to evaluate the presence and concentrations of antibodies specific for celiac disease (CeD) and antibodies related to inflammatory bowel diseases (IBD) in patients with RAS without gastrointestinal symptoms. Antibodies against tissue transglutaminase (anti-tTG), deaminated gliadin peptides (DGP), deaminated gliadinanalogous fragments (anti-GAF-3X) and Saccharomyces cerevisiae (ASCA) were determined by ELISA and antineutrophil cytoplasmic antibodies (ANCA) by indirect immunoflurescence (IIF) in 57 patients with RAS and 60 control subjects. The prevalence of CeD specific antibodies did not differ between RAS patients and controls. However, the concentrations of IgA anti-tTG, IgA anti-GAF-3X antibodies in patients with RAS were significantly higher compared to controls ( $\mathrm{p}=0.002$ and $\mathrm{p}=0.04$ respectively). Histological changes consistent with CeD were confirmed by duodenal biopsy in one RAS patient with highly positive IgA anti-tTG, anti-GAF-3X and antiDGP antibodies. Higher prevalence along with higher concentrations of IgG ASCA were found in RAS patients compared to controls ( $\mathrm{p}<0.01$ ). Patients with positive IgG ASCA in the absence of clinical symptoms decided not to pursue any further testing. Dysfunction of oral mucosa and the exposure to various antigens might be a reason for the loss of tolerance resulting in increased production of autoantibodies. It seems likely that antibodies are markers of aberrant immune response, rather than key effectors involved in the pathogenesis of the disease.
\end{abstract}

\section{Introduction}

Recurrent aphthous stomatitis (RAS) is an inflammatory disease of oral mucosa characterized by recurring episodes of solitary or multiple painful aphthous ulcers. The prevalence of RAS is approximately $20 \%$ in the general population (Jurge et al., 2006). The precise etiology of RAS is still uncertain, but it has been proven that a number of factors have direct influence on the RAS occurrence, including inheritance, stress, trauma, exposure to certain food, smoking cessation, nutritional deficiencies and hormonal imbalance. Local and systemic conditions, immunological and microbial factors may also have a pathogenic role (Chavan et al., 2012). RAS can be presented as primary, idiopathic condition and as a secondary RAS, which is associated with a systemic disease (Natah et al., 2004). The presence of refractory and recurrent oral aphthous ulcerations could imply the existence of underlying inflammatory disease, such as celiac or inflammatory bowel disease (Baccaglini et al., 2011; Lankarani et al., 2013).

Celiac disease $(\mathrm{CeD})$ is a chronic small intestinal immune-mediated enteropathy precipitated by exposure to dietary gluten in genetically predisposed individuals (Ludvigsson et al., 2013). CeD is characterized by the presence of autoantibodies, which are currently included in the definition and diagnostic guidelines for CeD (Murch et al., 2013). CeDspecific serologic markers, such as antibodies against tissue transglutaminase (tTG), endomysial antibodies (EMA), antibodies against deaminated gliadin peptides (DGP) and deaminated gliadin analogous fragments (GAF) are considered to be a reliable diagnostic tool for CeD. $\mathrm{CeD}$ can be presented with classical gastrointestinal symptoms, but also as an atypical, silent and latent form with a wide spectrum of non-

\footnotetext{
* Corresponding author.

E-mail address: borivojbijelic@yahoo.com (B. Bijelić).
} 
gastrointestinal symptoms. RAS may be one of the symptoms and it is present as the sole manifestation in $5 \%$ of CeD patients (Shakeri et al., 2009). On the other hand, the reported prevalence of CeD in RAS patients varies between 0\%-31\% (Shakeri et al., 2009; Ferguson et al., 1976; Campisi et al., 2008; Sedghizadeh et al., 2002; Yasar et al., 2012). The association between CeD and RAS has been evaluated in several studies, but the supporting evidence remains equivocal (Sedghizadeh et al., 2002; Yasar et al., 2012).

Inflammatory bowel diseases (IBD) including Crohn's disease (CD) and ulcerative colitis (UC) are chronic inflammatory diseases with primary intestinal involvement. IBD patients may exhibit a wide range of non-intestinal signs and symptoms, known as extraintestinal manifestations (EIM). Oral lesions can be the primary presenting signs preceding gastrointestinal symptoms in $5 \%-10 \%$ of affected patients (Trikudanathan et al., 2012). Although no specific direct association has been reported, it is assumed that $10 \%$ of patients with UC and $20 \%-30 \%$ of those with CD have RAS (Trikudanathan et al., 2012). Patients with IBD and other EIMs may suffer from RAS more often than others (Lankarni et al., 2013; Trikudanathan et al., 2012). The relevance of specific antibodies for IBD diagnosis is not so strong as for CeD. Serologic markers for UC and CD are antineutrophil cytoplasmic antibodies (ANCA) and antibodies against Saccharomyces cerevisiae (ASCA) respectively. So far there is no evidence of association between RAS and presence of these IBD-related antibodies.

Based on the presumed common etiopathogenesis of RAS, CeD and IBD, which includes dysregulated mucosal immunity, the aim of our study was to investigate the presence and concentrations of new and accepted CeD-specific and IBD-related serologic markers in patients with RAS free of gastrointestinal symptoms.

\section{Patients and methods}

\subsection{Patients}

The study group compromised 57 adult patients (35 males; median age $43.7 \pm 20.6$, range $18-76$ years) consecutively referred to the Clinic for Periodontology and Oral Medicine, School of Dental Medicine, University of Belgrade for management of recurrent mouth ulcers (between April 2012 and May 2015). All patients were diagnosed with minor RAS based on the accepted criteria (Jurge et al., 2006). Patients' consent was obtained for oral examination and for taking venous blood samples.

A general medical history was taken and clinical examination was performed on each patient. Other causes of recurrent oral ulceration such as: systemic diseases, trauma, hematinic deficiencies and HIV infection were ruled out. The exclusion criterion for Behçet's disease was the absence of genital ulcerations, ocular and cutaneous involvement. Also, the patients were not receiving regular treatment with drugs that have been associated with development of RAS (Chavan et al., 2012). A complete history was taken from all subjects regarding number of aphthous ulcers, duration and number of attacks per year.

The control group consisted of 60 subjects as follows: 30 age- and gender-matched healthy subjects that voluntarily accepted to participate in the study and 30 volunteer blood donors. Physical examination and complete oral cavity examination performed in a group of 30 healthy subjects revealed the absence of any abnormality. The part of the control group comprised of 30 volunteer blood donors was not examined for the presence of clinical symptoms of RAS. The purpose of the control groups was the establishment of accurate cut-off values for autoantibodies in our population.

The Ethics Committee of the School of Dental Medicine, University of Belgrade, Serbia, approved the study design, data collection methods and procedure for obtaining informed consent (Approval Number 36/ 13). The study was carried out according to the principles of the Declaration of Helsinki.

Laboratory examinations were performed in the Laboratory
Department in the Clinic of Allergy and Immunology, Clinical Center of Serbia, and in the Laboratory for Modification of Biological Response, Department of Experimental Oncology, Institute of Oncology and Radiology of Serbia.

Aliquots of the sera stored at $-20^{\circ} \mathrm{C}$ were used to detect antibody reactivity. Serum IgA level was measured to rule out IgA deficiency.

\subsection{Determination of CeD-specific antibodies}

IgA and IgG antibodies against human recombinant tissue transglutaminase (anti-tTG), and IgA antibodies against deaminated gliadin analogous fragments (GAF) were evaluated by ELISA according to the instructions of the manufacturer (Euroimmun AG, Luebeck, Germany). The anti-GAF-3X ELISA is based on a completely new antigen which reflects a peptide derived from native gliadin by deamidation through tTG. The syntetic DNA coding for GAF includes threefold repetitive modified copies of immunodominant nanopeptides cloned into a prokaryotic expression vector and expressed in E.coli. The cut-offs for positivity at 20 and 25 relative units per milliliter $(\mathrm{RU} / \mathrm{mL})$ for anti-tTG and anti-GAF-3X respectively, were used in accordance with the recommendations of the manufacturer.

IgA and IgG antibodies against deaminated gliadin peptides (DGP) were determined by commercial ELISA (BioSystems SA, Barcelona, Spain). According to recommendations of the manufacturer, samples with concentrations $\geq 10 \mathrm{U} / \mathrm{mL}$ were considered positive. Based on the results obtained from sera of volunteer blood donors and the healthy control group, a cut-off values (mean $+2 \mathrm{SD}$ ) were set at $12.5 \mathrm{U} / \mathrm{mL}$ for IgA and $26 \mathrm{U} / \mathrm{mL}$ for IgG anti-DGP antibodies.

\subsection{Determination of IBD-related antibodies}

IgA and IgG ASCA were detected in 40 RAS patients and 30 healthy controls by ELISA employing phosphopeptidomannan with a cut-off for positivity at $10 \mathrm{U} / \mathrm{mL}$ (Orgentec Diagnostica, Mainz, Germany), according to the manufacturer's instructions.

The presence, titers and staining pattern of IgG ANCA were detected in 57 patients with RAS and 60 controls using an indirect immunofluorescence (IIF) assay with ethanol-fixed preparations of neutrophils (Euroimmun AG, Luebeck, Germany), starting with 1:20 dilution. To determine the serum endpoint titers, sera were diluted up to the highest dilution that still gave the characteristic fluorescence pattern.

\subsection{Statistical analysis}

Statistical analyses were performed using SPSS 17.0 (SPPS Inc. Illinois, USA). The two-tailed, parametric $t$-test and non-parametric Chisquare and Mann-Whitney tests were used to test for statistically significant differences of independent samples in two groups. Correlations of antibody tests were assessed by Spearman's correlation coefficient. Values of $\mathrm{p}<0.05$ were considered significant.

\section{Results}

Demographic and clinical features of patients are presented at Table 1. Patients with RAS were clinically asymptomatic for CeD and IBD.

\subsection{Prevalence of autoantibodies in RAS patients and controls}

IgA or IgG anti-tTG positivity was present in $1 / 57$ (1.8\%) and IgA anti-GAF-3X in $2 / 56(3.6 \%)$ patients with RAS, which was not significantly different from the control subjects $(\mathrm{p}>0.05)$. Eleven RAS patients $(19.6 \%)$ were IgA and/or IgG anti-DGP positive comparing to five IgA-DGP (8.3\%) and eight IgG-DGP (13.3\%) positive subjects in the control group ( $\mathrm{p}=0.067$ and $\mathrm{p}=0.253$, respectively).

The prevalence rates of IgA ASCA seropositivity did not differ 
Table 1

Demographic and clinical characteristics of patients with recurrent aphthous stomatitis.

\begin{tabular}{ll}
\hline Demographic and diagnostic data & $\mathrm{n}=57$ \\
\hline Gender: F/M & $22 / 35$ \\
Age, years, mean \pm SD (range) & $43.7 \pm 20.6$ \\
Mean disease duration (years) mean (range) & $9.71 \pm 11.35(0.5-30)$ \\
Mean ulcer healing time & \\
$\leq 1$ week & $16(28.1 \%)$ \\
$>1$ week & $41(71.9 \%)$ \\
Frequency of attacks & $11(19.3 \%)$ \\
3-6 attacks per year & $46(80.7 \%)$ \\
$>6$ attacks per year & \\
Lesions at presentation & $12(21.1 \%)$ \\
Solitary & $45(78.9 \%)$ \\
Multiple &
\end{tabular}

between RAS patients and controls ( $3 / 40$ vs. $3 / 30$, p > 0.05). On the contrary, the frequency of IgG ASCA positivity was significantly higher in RAS patients compared to controls $(8 / 40$ vs. $0 / 30, p=0.009)$.

The prevalence of ANCA did not significantly differ between patients with RAS and controls. Namely, atypical ANCA were detected in three patients with RAS and one healthy subject. Titers of ANCA were low, ranged from 1:20 to $1: 80$.

\subsection{Concentration of autoantibodies in RAS patients and controls}

The concentrations of IgA anti-tTG and IgA anti-GAF-3X antibodies in patients with RAS were significantly higher compared to concentrations determined amongst healthy volunteers (Mann-Whitney test, $p=0.002$ and $p=0.04$ respectively) (Fig. 1 and Fig. 2).

There was also a significantly elevated concentration of IgG ASCA in patients with RAS compared to the control group (Mann-Whitney test, $\mathrm{p}=0.005$ ) (Fig. 3).

Levels of IgG and IgA anti-DGP antibodies, IgG anti-tTG and IgA ASCA were not significantly different in RAS patients compared to controls.

In patients with RAS IgA anti-tTG levels correlated significantly with IgA anti-GAF-3X levels (Spearman's coefficient of rank correlation $(\rho)=0.36, p=0.007)$. In contrast, IgA anti-DGP did not correlate with IgA tTG and IgA GAF-3X antibodies ( $p>0.05$ ).

One patient with highly positive antibodies against tTG (IgA), GAF3X (IgA) and DGP (IgA and IgG) in the absence of gastrointestinal symptoms was referred to a gastroenterologist. He underwent endoscopic biopsy of the duodenal mucosa. Histology findings were characteristic of $\mathrm{CeD}$, showing villous atrophy with crypt hyperplasia and increased intraepithelial lymphocytes (Marsh type III). A significant improvement of stomatitis was noted after two months of gluten-free diet. Aphthous lesions completely resolved after six months of gluten

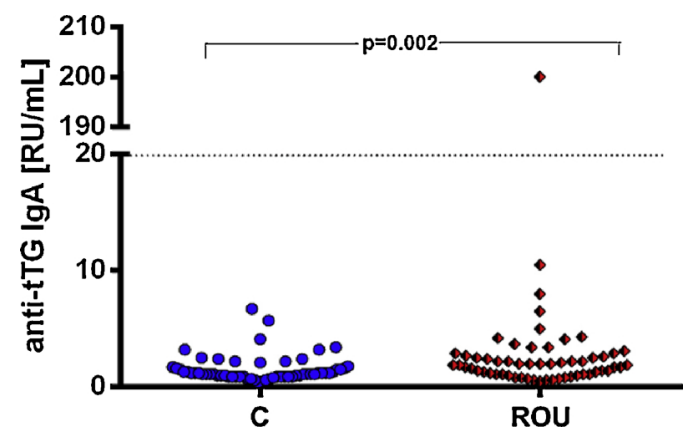

Fig. 1. IgA antibodies against tissue transglutaminase (tTG) in patients with recurrent aphtous stomatitis (RAS) and healthy controls (C). Dashed line indicates the cut-off for positivity.

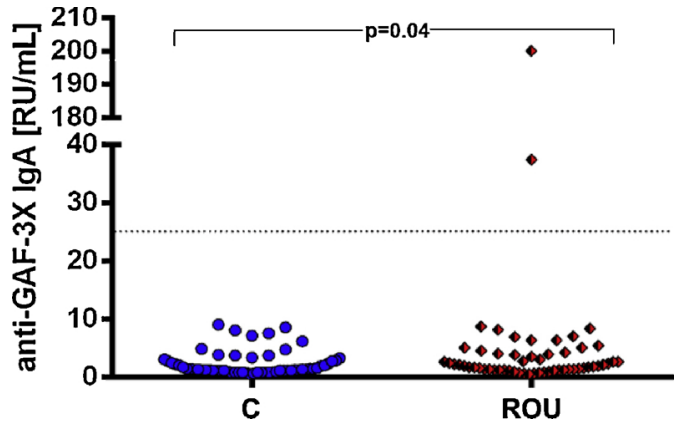

Fig. 2. IgA antibodies against deaminated gliadin analogous fragments (GAF) in patients with recurrent aphtous stomatitis (RAS) and healthy controls (C). Dashed line indicates the cut-off for positivity.

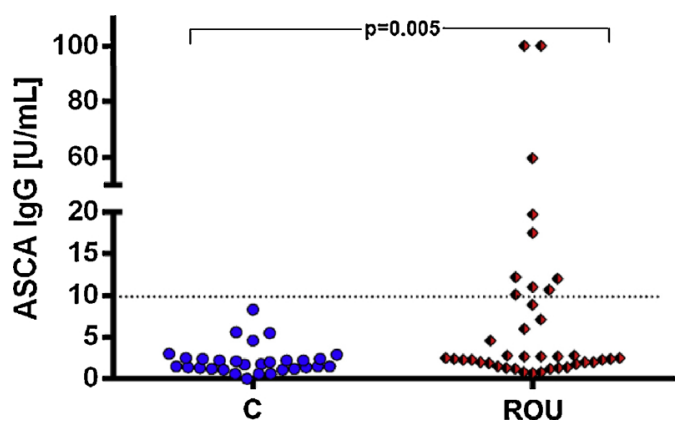

Fig. 3. IgG antibodies against Saccharomyces cerevisiae (ASCA) in patients with recurrent aphtous stomatitis (RAS) and healthy controls (C). Dashed line indicates the cut-off for positivity.

withdrawal.

For the three patients with high levels of ASCA IgG $(>50 \mathrm{U} / \mathrm{mL})$ free of gastrointestinal symptoms a recommended endoscopic examination was postponed due to their doubts and fears related to the procedure.

There were no significant associations between characteristics of RAS, including mean duration of disease, type of ulcers, frequency of attacks and number of lesions and concentrations of determined autoantibodies.

\section{Discussion}

It is assumed that immune-mediated mucosal damage in RAS can be induced by various predisposing environmental factors in genetically susceptible individuals. Oral mucosa, as a part of a gastrointestinal tract, participate in the creation of immune tolerance to orally introduced antigens, such as food and commensal microbes. It was reported that RAS represents an increased risk of underlying gastrointestinal immunoinflammatory diseases (Ferguson et al., 1976). Likewise, both $\mathrm{CeD}$ and IBD are related to inadequate mucosal immune response to food and commensal microbe antigens (Geremia et al., 2014; Sollid, 2002). It is believed that both CeD and IBD are exacerbated by or lead to an impairment of the intestinal barrier. However, pathophysiologic mechanism, as well as clinical and therapeutic approach, are quite distinct for these two gastrointestinal inflammatory conditions (Murch et al., 2013; Trikudanathan et al., 2012; Geremia et al., 2014; Sollid, 2002; Basso et al., 2014).

Anti-endomysial antibodies (EMA), anti-tTG antibodies, and antibodies against deaminated gliadin peptides are considered to be specific for CeD (Murch et al., 2013). The concentration of these antibodies has been mostly investigated in CeD patients, while in the RAS patients these CeD-specific antibodies have been tested only for their prevalence. Anti-GAF-3X antibody test based on specially designed immunodominant epitopes has been investigated in a few studies (de 
Chaisemartin et al., 2015), but none of them included RAS patients. It is our belief that this is the first study that examined anti-GAF-3X antibodies in patients with RAS. Schwertz et al. developed an ELISA based on gliadin-related nonapeptides as a highly sensitive and specific assay for diagnosis of celiac disease (Schwertz et al., 2004). The investigations of novel anti-GAF-3X IgA antibodies showed that they are more sensitive and specific than anti-gliadin IgA antibodies, as provided by the manufacturer.

In the present study, prevalence of IgA or IgG anti-tTG reactivity was $1.8 \%$ and IgA anti-GAF-3X was $3.6 \%$ in patients with RAS, which was not significantly different compared to control subjects. Our study detected one new case of $\mathrm{CeD}$, with severe histopathological findings in duodenal biopsy. The prevalence of $\mathrm{CeD}$ in our group of RAS patients was $1.75 \%$, compared to $0 \%$ in matched healthy controls and $1-2 \%$ in the general population worldwide. These data are in agreement with previous studies (Shakeri et al., 2009; Ferguson et al., 1976; Sedghizadeh et al., 2002; Yasar et al., 2012). Although our study found no significant prevalence of the investigated serologic markers in RAS patients, we have found statistically higher concentrations of IgA antitTG and anti-GAF-3X antibodies, which might require further consideration. Disruption of epithelial cell junctions and enhanced gluten penetration can lead to production of antibodies against deaminated gluten peptides in early stage of $\mathrm{CeD}$, even before a significant mucosal damage develops (Rauhavirta et al., 2014). As a marker of small bowel mucosal damage, anti-tTG antibody levels correlate with pathological findings in CeD patients (Donaldson et al., 2007), but still, anti-tTG antibodies could be low or even negative in adult patients with mild CeD (Sollid, 2002). Therefore, a negative anti-tTG antibodies in RAS patients can not rule out the coexistence of latent or mild form of CeD.

We also found a significantly higher prevalence and elevated concentration of IgG ASCA in patients with RAS compared to the control group. It has been established that patients with $C D$ and UC are frequently positive for ASCA and ANCA, respectively. Antibodies to baker's yeast and brewer's yeast (ASCA) have been described in up to 65\% of patients with CD (Basso et al., 2014). It has been demonstrated that the specific antigen is a mannan localized in the yeast cell wall. The pathogenic role of ASCAs in CD is not completely understood, but one hypothesis links them to increased intestinal permeability (Krause and Milo, 2004). Due to this presumed break in the epithelial integrity, increased exposure of the mucosa to common food antigens, such as yeasts, may result in an exaggerated antibody response.

Besides intestinal inflammatory conditions, a significantly higher level of IgG ASCA was discovered in patients with Behçet's disease (BD) by Krause et al. (Krause et al., 2002). Aforementioned study and another study (Rhee et al., 2005) used RAS patients as a control group and found high level of ASCA in 1/10 and 1/12 RAS patients, respectively. Moreover, these authors also found positive correlation between titers of IgG ASCA with the number of aphthae episodes in a year in healthy relatives of BD patients who have RAS (Monselise et al., 2006). On the other hand, ASCA had been found in overt CeD, as well as in early stage of $\mathrm{CeD}$, together with some other anti-microbial antibodies, suggesting a dysregulated immune response to food and bacterial antigens. Interestingly, anti-microbial antibodies and ASCA showed a decrease after implementation of a gluten-free diet (Viitasalo et al., 2014; Conrad et al., 2002). Conversely, a recent study found significantly increased positivity for anti-tTG and anti-gliadin antibodies in patients with IBD compared to controls, even if CeD could not be confirmed by histology in any patient (Watanabe et al., 2014).

Although recent studies have suggested that imbalances in the oral microbiota may contribute to the etiopathogenesis of RAS, no specific bacterial species associated with RAS have been identified (Hijazi et al., 2015). The latest findings confirm that certain bacteria species were represented more frequently on the oral mucosa of patients with BD and RAS, as compared to healthy controls (Seoudi et al., 2015). These findings may or may not be linked to the similarities in ASCA production in BD and RAS patients, but certainly indicate that mucosal microbiome changes may play a role in the etiology of chronic mucosal inflammatory conditions.

It is well established that oral and gut mucosal immune system constitute an effective and dynamic network in which numerous types of cells and molecules contribute to the balance between immune tolerance and immune response (Wu et al., 2014). Oral mucosa is the first barrier of digestive system. Mucosal barrier dysfunction and the exposure to various antigens can lead to modified mucosal immunity and loss of humoral tolerance to self and microbiota antigens.

The presence of several types of antibodies in the sera of RAS patients suggests an immune mediated nature of RAS. If nothing other, increased levels of antibodies against gliadin, t-TG and yeasts in patients with RAS might result from a common tight junction defects of mucosal barrier similar to those already described in patients with CeD and IBD (Fasano, 2012). It seems likely that antibodies are markers of aberrant immune response, rather than key effectors involved in the pathogenesis of the disease.

The low prevalence of $\mathrm{CeD}$ and IBD associated antibodies implies that their determination is not generally recommended for all patients with RAS. On the other hand, if the likelihood of gastrointestinal disease is high or aphthous stomatitis is unresponsive to conventional treatment these tests would be useful (Shakeri et al., 2009).

Evaluation of the possible clinical significance of our findings and elucidation of the cause-effect relationship and interactions between CeD specific and IBD related antibodies and patients with RAS requires a further long term follow up investigations and analysis of prospectively collected serum samples from a large cohort of patients with RAS.

\section{Author contributions}

All the authors have accepted responsibility for the entire content of this submitted manuscript and approved submission.

\section{Research funding}

The authors are grateful to the Ministry of Education, Science and Technological Development of the Republic of Serbia for the financial support (Project 175011).

\section{Declaration of interest}

None.

\section{Acknowledgments}

The authors would like to thank Tatjana Petrović for her excellent technical assistance. We thank all patients and healthy volunteers for contributing to this study.

\section{References}

Baccaglini, L., Lalla, R.V., Bruce, A.J., Sartori-Valinotti, J.C., Latortue, M.C., Carrozzo, M., Rogers 3rd, R.S., 2011. Urban legends: recurrent aphthous stomatitis. Oral Dis. 17, 755-770.

Basso, D., Zambon, C.F., Plebani, M., 2014. Inflammatory bowel diseases: from pathogenesis to laboratory testing. Clin. Chem. Lab. Med. 52, 471-481.

Campisi, G., Di Liberto, C., Carroccio, A., Compilato, D., Iacono, G., Procaccini, M., et al., 2008. Coeliac disease: oral ulcer prevalence, assessment of risk and association with gluten-free diet in children. Dig. Liver Dis. 40, 104-107.

Chavan, M., Jain, H., Diwan, N., Khedkar, S., Shete, A., Durkar, S., 2012. Recurrent aphtous stomatitis: a review. J. Oral Pathol. Med. 41, 577-583.

Conrad, K., Schmechta, H., Klafki, A., Lobeck, G., Uhlig, H.H., Gerdi, S., Henker, J., 2002. Serological differentiation of inflammatory bowel diseases. Eur. J. Gastroenterol. Hepatol. 14, 129-135.

de Chaisemartin, L., Meatchi, T., Malmut, G., Fernani-Oukil, F., Hosking, F., Rault, D. et al., 2015. Application of deamidated gliadin antibodies in the follow-up of treated celiac disease. PLoS One 10, e0136745.

Donaldson, M.R., Firth, S.D., Wimpee, H., Leiferman, K.M., Zone, J.J., Horsley, W., et al., 2007. Correlation of duodenal histology with tissue transglutaminase and endomysial 
antibody levels in pediatric celiac disease. Clin. Gastroenterol. Hepatol. 5, 567-573. Fasano, A., 2012. Leaky gut and autoimmune diseases. Clin. Rev. Allergy Immunol. 42, 71-78.

Ferguson, R., Basu, M.K., Asquith, P., Cooke, W.T., 1976. Jejunal mucosal abnormalities in patients with recurrent aphthous ulceration. Br. Med. J. 1, 11-13.

Geremia, A., Biancheri, P., Allan, P., Corazza, G.R., Di Sabatino, A., 2014. Innate and adaptive immunity in inflammatory bowel disease. Autoimmun. Rev. 13, 3-10.

Hijazi, K., Lowe, T., Meharg, C., Berry, S.H., Foley, J., Hold, G.L., 2015. Mucosal microbiome in patients with recurrent aphtous stomatitis. J. Dent. Res. 94, 87S-94S.

Jurge, S., Kuffer, R., Scully, C., Porte, S.R., 2006. Mucosal disease series. Number VI. Recurrent aphtous stomatitis. Oral Dis. 12, 1-21.

Krause, I., Milo, G., 2004. Anti-Saccharomyces cerevisiae antibodies and autoimmune diseases. In: Shoenfeld, Y., Rose, N.R. (Eds.), Infection and Autoimmunity. Elsevier, pp. 467-470.

Krause, I., Monselise, Y., Milo, G., Weinberger, A., 2002. Anti-Saccharomyces cerevisiae antibodies: a novel serologic marker for Behçet's disease. Clin. Exp. Rheumatol. 20, 21-24.

Lankarani, K., Sivandzadeh, G.R., Hassanpour, S., 2013. Oral manifestation in inflamatory bowel disease: a review. World J. Gastroenterol. 19, 8571-8579.

Ludvigsson, J.F., Leffler, D.A., Bai, J.C., Biagi, F., Fasano, A., Green, P.H., et al., 2013. The Oslo definitions for coeliac disease and related terms. Gut 62 (1), 43-52.

Monselise, A., Weinberger, A., Monselise, Y., Faser, A., Sulkes, J., Krause, I., 2006. AntiSaccharomyces cervisiae antibodies in Behçet's disease -a familiar study. Clin. Exp. Rheumatol. 24, 87-90.

Murch, S., Jenkins, H., Auth, M., Bremner, R., Butt, A., France, S., et al., 2013. Joint BSPGHAN and Coeliac UK guidelines for the diagnosis and management of coeliac disease in children. Arch. Dis. Child. 98, 806-811.

Natah, S.S., Konttinen, Y.T., Enattah, N.S., Ashmmakhi, N., Sharkey, K.A., HayrinenImmonen, E., 2004. Recurrent aphthous ulcers today: a review of the growing knowledge. Int. J. Oral Maxillofac. Surg. 33, 22-34.

Rauhavirta, T., Lindfors, K., Koskinen, O., Laurila, K., Kurppa, K., Saavalainen, P., et al.,
2014. Impaired epithelial integrity in duodenal mucosa in early stages of celiac disease. Transl. Res. 164, 223-231.

Rhee, S.-H., Kim, Y.-B., Lee, E.-S., 2005. Comparison of Behçet's disease and recurrent aphtous ulcer according to characteristics of gastrointestinal symptoms. J. Korean Med. Sci. 20, 971-976.

Schwertz, E., Kahlenberg, F., Sack, U., Richter, T., Stern, M., Conrad, K., et al., 2004. Serologic assay based on gliadin-related nonapeptides as a highly sensitive and specific diagnostic aid in celiac disease. Clin. Chem. 50 (12), 2370-2375.

Sedghizadeh, P.P., Shuler, C.F., Allen, C.M., Beck, F.M., Kalmar, J.R., 2002. Celiac disease and recurrent aphthous stomatitis: a report and review of the literature. Oral Surg. Oral Med. Oral Pathol. Oral Radiol. Endod. 94, 474-478.

Seoudi, N., Bergmeier, L.A., Drobniewski, F., Paster, B., Fortune, F., 2015. The oral mucosal and salivary microbial community of Behcet's syndrome and recurrent aphtous stomatitis. J. Oral Microbiol. 7, 27150.

Shakeri, R., Zamani, F., Sotoudehmanesh, R., Amiri, A., Mohamadnejad, M., Davatchi, F., et al., 2009. Gluten sensitivity enteropathy in patients with recurrent aphthous stomatitis. BMC Gastroenterol. 9, 44.

Sollid, L.M., 2002. Coeliac disease: dissecting a complex inflammatory disorder. Nat. Rev. Immunol. 2, 647-655.

Trikudanathan, G., Venkatesh, P.G., Navaneethan, U., 2012. Diagnosis and therapeutic management of extra-intestinal manifestations of inflammatory bowel disease. Drugs 72, 2333-2349.

Viitasalo, L., Niemi, L., Ashorn, M., Ashorn, S., Braun, J., Huhtala, H., et al., 2014. Early microbial markers of celiac disease. J. Clin. Gastroenterol. 48, 620-624.

Watanabe, C., Komoto, S., Hokari, R., Kurihara, C., Okada, Y., Hozumi, H., et al., 2014. Prevalence of serum celiac antibody in patients with IBD in Japan. J. Gastroenterol. 49, 825-834.

Wu, R.Q., Zhang, D.F., Chen, Q.M., Chen, W., 2014. The mucosal immune system in the oral cavity-an orchestra of T cell diversity. Int. J. Oral Sci. 6, 125-132.

Yasar, S., Yasar, B., Abut, E., Asirian Serdar, Z., 2012. Clinical importance of celiac disease in patients with reccurrent aphtous stomatitis. Turk. J. Gastroenterol. 23, 14-18. 